\title{
PC-based apparatus for characterising high frequency magnetic cores
}

\author{
S.-C.Wang and C.-L.Chen
}

\begin{abstract}
A PC-based automatic characterisation system (ACS) has been developed to automatically characterise magnetic materials under square wave cyclic excitation from $1 \mathrm{kHz}$ to $100 \mathrm{kHz}$. A computer-controlled $\mathrm{AC}$ to $\mathrm{AC}$ converter has been developed for the excitation circuit. A digital oscilloscope gathers the required data through the 8255 interface card. The crrors in the ACS have been analysed and overall repeatability is better than $\pm 0.35 \%$. The system has the advantages of ease of use, absencc of long-term measuring system drifts and ready variation of high induction and high frequency test parameters. The system operates automatically and measurements on three commercial core materials are reported. Comparisons with the data of manufacturers are included.
\end{abstract}

\section{Introduction}

Precise characteristics of the magnetic components are essential for the design of electromagnetic devices. In order to deliver the maximum power and obtain smaller and lighter circuits, the operating frequencies are being pushed higher. When the operating frequency is increased, the volume and weight of the circuit components will be reduced, and the heatsink area is also reduced along with the volume reduction. However, the switching loss of the components will increase owing to the frequency increase. Direct characterisation of the magnetic behaviour is difficult because of the magnetic flux inside the core. Hall effect sensors may be inserted into the core to carry out measurements. However, this is expensive and impractical for the designers. A conventional method [1] utilised a series R-C circuit to integrate the induced electromotive force (EMF) into proportional magnetic flux. An oscilloscope captures the exciting current and flux. Then the hysteresis loop is presented in the $X . Y$ operation mode. This method requires wide bandwidth oscilloscope and good phase compensation to prevent excessive phase shift, which will result in incorrect loop display. Microprocessor-based measurements [2-5] are proposed to characterise magnetic cores. The fast analogue-to-digital converters (ADCs) are used as data acquisition devices in these approaches. The ADCs sample and digitise the primary excitation current and the sccondary induced EMF. The acquired data are manipulated in microprocessors by numcrical integration and process algorithms. These techniques have been used for many years to obtain core data at lower frequencies, but their accuracy and validity become questionable while they are used at higher frequencies. The increasing use of power clectronic devices has prompted accurate measurements of magnetic materials at the higher frequencies.

\footnotetext{
() IEE, 1999

IEE Proceedings online no. 19990527

DOI: 10. 1049/ip-smt:19990527

Paper first received 26th October 1998 and in revised form 30th $\Lambda$ pril 1999

S.-C. Wang is with the Department of Elextrical Engineering, Kuang Wu Institute of Technology and Commerce, Pcito, Taipei 1 I 202, Taiwan

C.-L. Chen is with the Department of Electrical Enginecring, National Taiwan
} Univessity, Tripei 10764, Taiwan
The core data provided by the manufacturers under high frequency and large induction is not sufficient. These data are necessary for the engineers to design inductors and transformers for switching circuits. The reasons for the insufficiency of core data are as follows. (i) The characterisation system cannot supply high frequency and high-level excitation sources. (ii) There is a lack of a systematic and flexible measurement procedure. (iii) The behaviour of core varies drastically due to the exciting waveforms, opcrating frequency, and temperature. Few papers have focused on the measurements of high frequency cores by using switching power conditions. Boglietti et al. [6] present a direct method for measuring iron losses under six-step square and sinusoidal excitation. The comparison may provide a useful indication for the electromagnetic device designers. Chen [7] pointed out an anomalous result, the core loss under square wave excitation is lower than for sine wave excitation as the operating frequency and maximum flux density are maintained constant.

A computcr-aided automatic characterisation system (ACS) has been devcloped to characterise magnetic cores under high frequency and high level induction. It can obtain core data systematically through a flexible measurement procedure. A personal computer (PC) serves as the system controller. It takes charge of the management and controls all the operations inside the ACS. A menu-driven user friendly interface software is developed to support this system. It interfaces with user and offers flexibility and ease of use. With the assistance of the ACS, the original characterisation work is transferred to the system.

\section{Theoretical basis}

Many parameters can be used to characterise a magnetic core. Those of greatest interest for power electronic application are the hysteresis loop and the corc loss. The arca of hysteresis loop is equal to the energy loss of a core. The hysteresis loop also includes additional information, such as the residual flux density, coercive force, and permeability. The measurement of the magnetic flux density $B$ and magnetic field intensity $H$ is the starting point for the investigation of core characteristic. 


\subsection{Investigation of hysteresis loops}

The hysteresis loop is displayed by the magnetic flux density and magnetic field intensity in the $B-I I$ plane. From Ampere's law, the magnetising current $\left(i_{m}\right)$ and $H$ in a wound core is given by

$$
H(t)=\frac{N_{p} i_{m}(t)}{l_{e}}
$$

where $N_{p}$ is the number of primary turns and $I_{c}$ is the core average length. The induced EMF $e(t)$ of the secondary winding, from Faraday's law, can be expressed by

$$
e(t)=-N_{s} \frac{d \Phi(t)}{d t}
$$

where $N_{s}$ is the number of secondary turns.

From eqn. 2, the magnetic flux can be obtained by

$$
\Phi(t)=\Phi_{0}-\frac{1}{N_{s}} \int_{t_{0}}^{t} c(t) d t
$$

where $\Phi_{0}$ is the flux at time $t_{0}$. The magnetic flux divided by the effective corc arca $A_{e}$ is $B(t)$. Therefore, the final form of $B(t)$ is

$$
B(t)=\frac{\Phi_{0}}{A_{e}}-\frac{1}{A_{e} N_{s}} \int_{i_{0}}^{t} e(t) d t
$$

The data on the magnetising current and induced EMF, captured under an exciting cycle, are enough to determine $H(t)$ and $B(t)$ by mcans of numerical processing algorithms [8]. Then the dynamic hysteresis loop can be displayed in the $B-H$ plane.

\subsection{Evaluation of core loss}

The core loss generally is separated into hysteresis loss and eddy current loss. The hysteresis loss is caused by the multivalued nature of the hysteresis loop. It is normally proportional to the operating frequency and the square of maximum magnetic flux density. The eddy current loss is induced by the effect of domain wall motion as a time-varying magnetic field is applied to the core. It is normally proportional to the square of the operating frequency and maximum flux density. The mathematical model of the core loss, from the Poynting's theorem, is given by eqn. 5 .

$$
P=\frac{1}{T} \int_{V_{e}} \oint H \cdot d B \cdot d V_{e}+\frac{1}{T} \int_{0}^{T} \int_{V_{e}} \frac{J^{2}}{\sigma} \cdot d V_{e} \cdot d t
$$

where $V_{e}$ and $\sigma$ are the effective volume and conductivity of the core, respectively. $T$ is the period of the excitation current. Since the two losses are related to the frequency and flux density, the other empirical equation [1] is given by

$$
P=k V_{c} f^{\eta} B_{m}^{\xi}
$$

where $\kappa, \eta$, and $\zeta$ are constant, their valuc is related to the physical characteristic of the core material.

For practical purposes, the methods already mentioned are not suitable for application to power electronic design. A measurement and numerical processing approach is used in this system. According to the energy conservation, the power flow into the core should be equal to the energy loss because the current of the secondary winding is zero. The power $P$ fed to the core under a cycle is given by eqn. 7 .

$$
P=\frac{1}{T} \int_{0}^{T} e(t) \cdot i_{m}(t) d t
$$

Substituting eqns. 1 and 2 into eqn. 7 yiclds the result:

$$
P=\frac{A_{e} l_{c}}{T} \int_{0}^{T} H \cdot \frac{d B}{d t} d t
$$

If $N_{p}=N_{s}$, and $A_{e} l_{e}=V_{e}$, the resulting core loss under per unit volume per cycle can be expressed as

$$
P=\frac{1}{T} \oint H \cdot d B
$$

In this way, the determination of the core loss can bc directly obtained from the dynamic hysteresis loop under a fixed measurement procedure. In addition, this method is applicable to the arbitrary periodic exciting waveforms.

\section{$3 \quad$ Measuring apparatus}

\subsection{Hardware architecture}

The hardware architecture of the ACS consists of a personal computer (PC) with an $8255 \mathrm{I} / \mathrm{O}$ card, an exciting power generator (EPG), a digital oscilloscope, a command control circuit (CCC), a current monitor circuit (CMC), and the core under test (CUT). Fig. 1 illustrates the connection of these devices. Principal functions of each unit are described as follows.

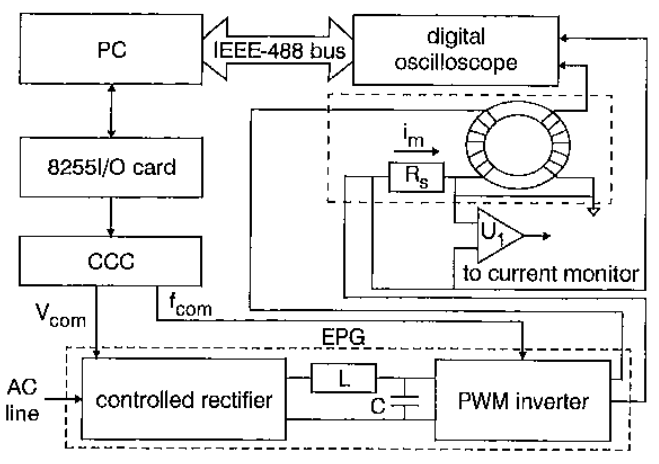

Fig.1 Harhare system architectare

3.1.1 Personal computer (PC): A PC with a fricndly user interface serves as the system controller. It takes charge of the management of the system operation and controls all the characterisation operations inside the system. With the aid of adaptive man-machine software, the characterisation procedures can be casily prepared and efficiently controlled. The PC sends specified voltage and frequency commands to drive the EPG through the command control circuit; the EPG then generates the excitation condition that is fed to the core under characterisation. The digital oscilloscope measures the responses of the CUT, and the acquired data are transmitted back to the PC through the IEEE-488 bus to determine the $B$ and $H$, display the hysteresis loop, and calculate the core loss. The closed-loop control provides flexibility for the various cores without alteration and achieves the automatic measurement.

3.1.2 Exciting power generator (EPG): The EPG is a computer-controlled $\mathrm{AC}$ to $\mathrm{AC}$ converter [9]. It develops a progtammable environment that provides the variable voltage variable frequency exciting power condition to characterise the core under test. The EPG is composed of a controlled rectificr and a PWM inverter, as shown in Fig. 2. The semi-bridge controlled rectifier converts the AC line into adjustable $\mathrm{DC}$ voltage under cosine mode control. 
The half-bridge PWM inverter switches the DC voltage to the specified exciting source with controllable frequency. Without online microprocessor computation or discrete circuit implementation, the EPG features quick output response and low control complexity. The output rating of the EPG is $1.2 \mathrm{~kW}$ with frequency range adjustable from $1 \mathrm{kHz}$, to $100 \mathrm{kHz}$.

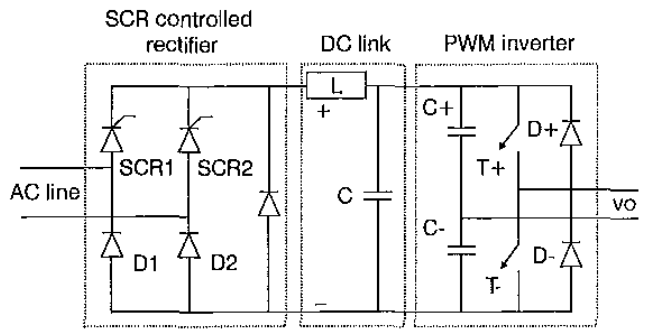

Fig.2 Exciting power gencrutor ( $E P G)$

3.1.3 Digital oscilloscope: Instcad of the discrete amplifiers and $\Lambda \mathrm{DCs}$, a digital oscilloscope is used as the data acquisition device to capture the data of the magnetising current and induced EMF. The acquired data are transmitted to the PC through the IEEE-488 bus. The oscilloscope features high sampling rate and bandwidth, so it is adequate to measure high frequency signals. With the digitising performance, the errors introduced by analogue integration are avoided. The captured data can be stored in memory for further process.

3.1.4 Command control circuit (CCC): The CCC, composed of digital to analogue converters (DACs) and amplificrs, accepts voltage/frequency commands from the PC to drive the EPG. It is the interface between the 8255 I/O port and the EPG.

3.1.5 Current monitor circuit (CMC): $\Lambda$ shunt current monitor provides a ground-reference voltage output to follow the magnitude and phase of the magnetisation current. The CMC provides a real-time correction to minimise the inevitable phase shift caused by the parasitic behaviour of the measurement setup, and prevent the EPG from short circuiting due to the core saturation.

\subsection{Software development}

The software developed in this system has the following purposes: to define characterisation procedures; to drive the connected instruments; to be user-friendly and easy to use; and to produce and manage the test results. It adopts an object-oriented programming (OOP) mochanism to develop a pull-down menu-driven user interface, and guarantees high-accuracy measurement results because of the imbedded adaptation functions in the procedure control. The characterisation procedure is facilitated by an OOP with enhanced graphic capability. It provides a flexible and userfriendly environment for system management.

3.2.1 User interface: The user interface is developed for both experienced and inexperienced computer users. It is designed to help the operator throughout the whole measurement process in a quite friendly way. There are two main objectives in the design process: to easily prepare charactcrisation procedures and manage test results, and to provide a user-friendly man-machine interface. These features can be implemented by customising the hardware platform via a suitable software package. Machine information processing is employed in the user interface to enhance the capability of the ACS. A high degrce of flexibility is required, and a repeatable function is supported to fit various characterisation conditions by introducing as few changes as possible in the man-machine interface, Visual Basic (VB) was used to develop the interface software and procedurc control program. VB is an object-oriented application framework for an MS-Windows program. A friendly user interface can be easily created in the VB intcgrated designing environment.

3.2.2 Software features: The characterisation software provides the capability of characterising the different types magnetic cores. All object clements are utilised for the integrated development environment by the OOP concepts. The software is very flexible, because the operator can define all the charactcrisation information and parameter input. The measurement results can be stored, edited, or deleted by using the powerful editor. The concept of the external procedure control obtains the benefits of easy operation and module programming, it is also helpful in maintenance, expandability, and further development.

Table 1: Parameters of the cores under test

\begin{tabular}{llllll}
\hline \multirow{2}{*}{ Type } & \multirow{2}{*}{ Material } & \multicolumn{3}{l}{ Dimensions } \\
& & $l_{e}(\mathrm{~cm})$ & $A_{e}\left(\mathrm{~cm}^{2}\right)$ & $V_{e}\left(\mathrm{~cm}^{3}\right)$ & $W(\mathrm{~g})$ \\
\hline TDK El-40 & H7C1 & 7.7 & 1.48 & 11.3 & 50.3 \\
TDK T5-10-2.5 & HP3000 & 2.18 & 0.0601 & 0.131 & 0.71 \\
Ferroxcube & $3 \mathrm{C} 8$ & 8.93 & 1.21 & 10.8 & 57.0 \\
\hline
\end{tabular}

\section{Experimental results}

The measurements on three commercial available cores are given to verify the performance of the ACS and the comparisons with the data of manufacturers are included. Shown in Table 1 are the parameters of the test cores. The characterisation results of the $\mathrm{H} 7 \mathrm{Cl}$ EI-40 ferrite core under $30 \mathrm{kHz}$ excitation is shown in Fig. 3 . The screen is the main menu of the user interface. The "input/output conditions' is used to input core data. The 'output panel' records the measurement results and the oscilloscope scale. The 'E-I curve' displays the waveforms of the magnetising current and induced EMF. The core characteristics obtained at fixed frequency but under different induction level for the T5-10-2.5 core are shown in Fig. 4. Shown in Fig. 5 is the plot of corc loss as a function of frequency for the $\mathrm{H} 7 \mathrm{C} 1$ corc under $0.2 \mathrm{~T}$ and $0.4 \mathrm{~T}$ induction, respectively. The core loss caused by the eddy current for ferrite material becomes significant when the exciting frequency is increased. The plot of the core loss as the function of the maximum induction for $3 \mathrm{C} 8$ under $20 \mathrm{kH} \%, 60 \mathrm{kHz}$, and $100 \mathrm{kHz}$ excitation frequencies is illustrated in Fig. 6.

The higher frequency features the larger loop area and results in the higher total core loss. The experimental results and fitting curves indicate that the core loss is proportional to the exciting frequency and the square of the peak flux density. This result is in agreement with the theoretical derivation as mention in Section 2. Illustrated in Fig. 7 is the comparison of the core loss obtained in this system with the data of manufacturer for the $\mathrm{H} 7 \mathrm{Cl}$ core. Obviously, the core loss obtained in the ACS is less than the data provided by the manufacturer under the test frequency range. The measurement results satisfactorily prove that the performances of the proposed apparatus are very adequate to characterise the magnetic materials for power electronic design. Moreover, the shape of the test core does not affect the results of the measurements.

IWE Proe-Su. Meas, Technol, Vol. 146, No. 6, November 1999 


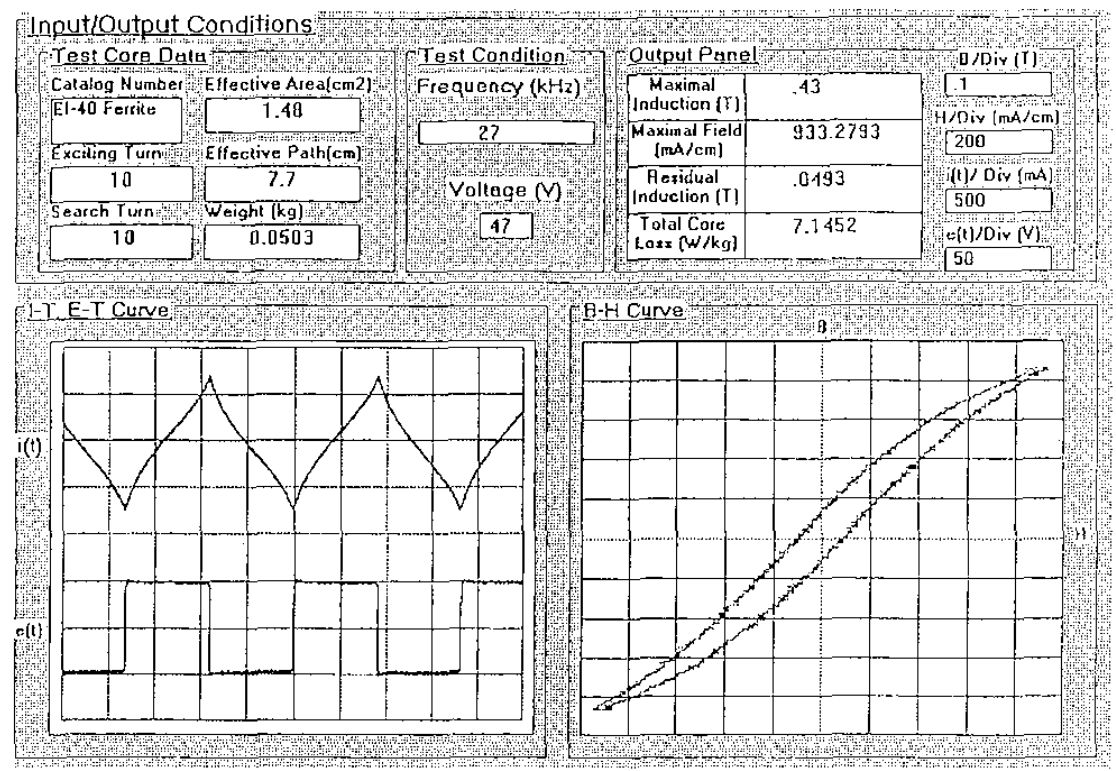

Fig.3 Plot of hysteresis loop for TDK II7CI El-40 ferite core

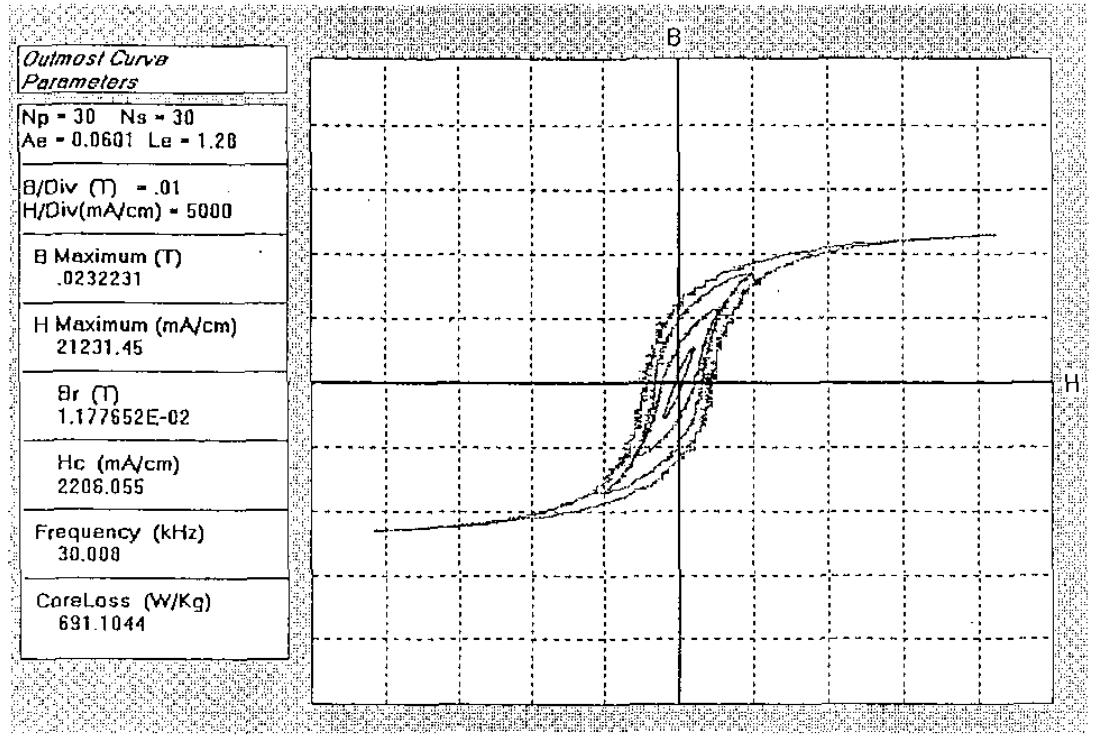

Fig.4 Plot of hysteresis loops for IIP3(XO) T5-10-2.5 core under different excitution indiction levels

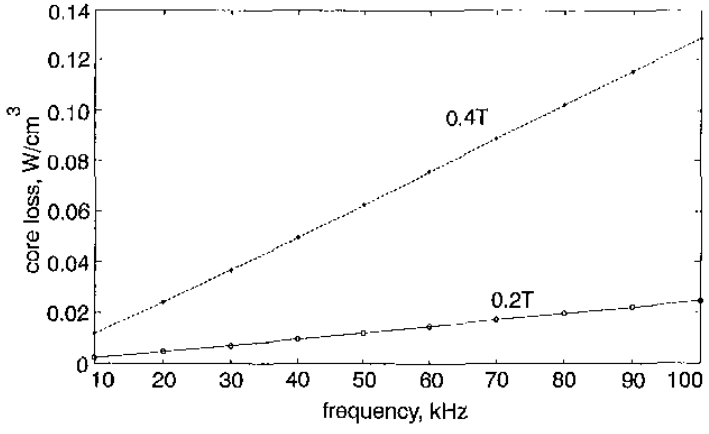
Fig. 5 Plot of core loss as finction of frequency for $1.7 \mathrm{Cl}$ under $0.2 \mathrm{~T}$ and

IEE Proc--Sci. Meas. Tectmol, Vol. 146, No. 6, Noventber 1999

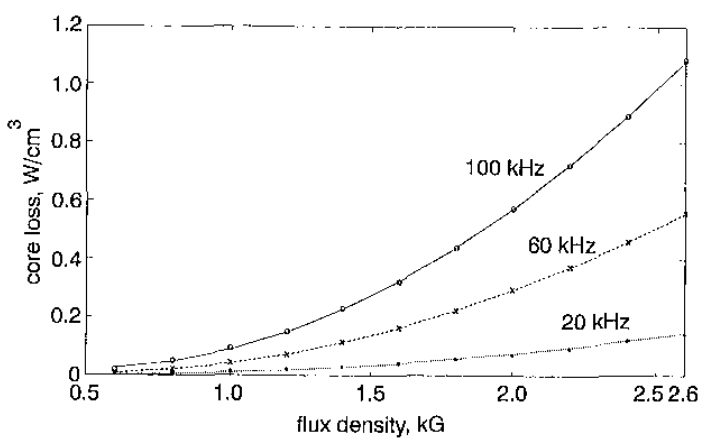

Fig.6 Plot of core has as finction of meximum indection for $3 C 8$ ander different firequencies 


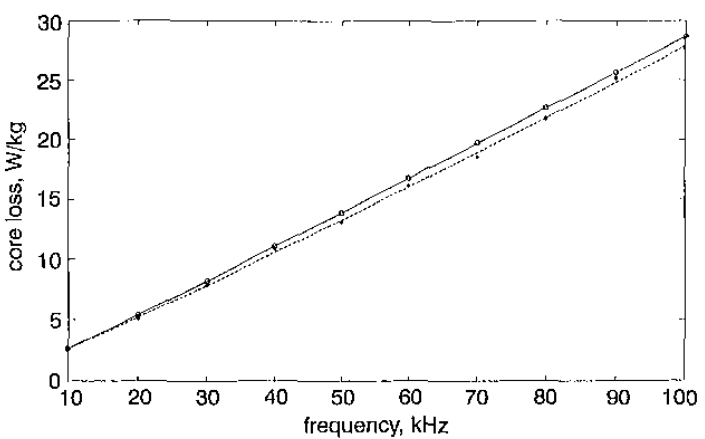

Fig. 7 Comparison of core loss obtuined in this system with dato of manufac-

data supplied by manufacture

* valıes obtained by proposed method

\section{Sensitivity and accuracy}

The sensitivity of the proposed method can be theoretically evaluated from the resolution and sampling rate of the data acquisition system. The minimum flux variation can be obtained from the product of the minimum EMF variation and the sampling rate of the oscilloscope. The theoretical sensitivity $S$ is given by

$$
S=\frac{V_{F S}^{r}}{2^{n} f_{s}}
$$

Where $n$ and $V_{I T S}$ are the bit number and full-scale voltage of the ADC, respectively, and $f_{s}$ is the sampling frequency. In this system, $n$ is 8 bits, $V_{\text {rs }}$ is equal to $10 \mathrm{~V}$, and the maximum sampling frequency is $10 \mathrm{GHz}$. Therefore, the maximum sensitivity is approximately $3.9 \times 10^{-12} \mathrm{~Wb}$. The measurement accuracy of the magnetic flux is evaluated by comparing the inductance of the CUT with a standard inductor. The maximum deviation between the measurement values and the standard values is less than $\pm 0.12 \%$.

The high performance oscilloscope improves the crrors introduced by the poor bandwidth, the trigger jitters, and the time delay. The short-term instability of the EPG can be ignored due to the good transient response. The resistor with low time constant and phase-shift correction procedure improves the phase-shift deviations caused by the poor frequency response of the current sensor and the parasitic capacitance of the measurement setup. The errors contributed by the DC offset of the ADC and numerical integration algorithm can be limited to a feasible tolerance by using alternate excitation, over sample, and a decimated technique. The high sensitivity enables more accurate core loss measurements, especially on the low-loss low-permeability cores. The overall repeatability of the ACS is better than $\pm 0.35 \%$.

\section{Conclusions}

A PC-based automatic measurement system for power electronic application to characterise high frequency magnetic cores has been developed. With the computer-based automation, a large portion of the original characterisation work is transferred to the system. The experimental tests give satisfactory results and illustrate the effectiveness of the prototype system.

This system demonstrates how a computer-aided characterisation system can be used to promote quality and efficicncy. It is effective in reducing the necessary manpower, as well as cost, and increasing the precision and repeatability of the characterisation job. The ACS is accurate because it agrees with the prescribed tolcrances. It is friendly since it is casy to use.

In comparison with the traditional method, the studicd system offers the following advantages:

- A computer controlled exciting power supply directly provides the high frequency and high-level induction conditions.

- Without magnetic transducers being necessary, the core characteristics can be obtained by measuring the magnetising current and induced EMF.

- Under a fixcd procedure, the ACS can obtain a large amount of core data.

- The PC-based method features a high degree of accuracy, easy operation, short measuring time, and good documentation.

\section{References}

1 McLYMAN, W.T.: 'Transformer and inductor design handbook' (Marcel Dekker, Inc., New York, 1978)

2 SANKARAN, P., KUMAR, V.I., and MURTI, G.K.: 'A microprocessor based measuring instrument for rapid testing of transformer corc material', IEEE Trans. 'ower Deliv., 1986, PWRD-1, pp. 19-21

3 BRANDOLINI, A., CARMINATI, F., and FERRERO, $\Lambda$. ' 'A completcly microprocessor-based measurement of magnetic propertics'. Proceedings of IMAC'84, Tokyo, Japan, 1984, pp. 137-142

4 CARMINATI, E., and FERRERO, $\Lambda$.: 'A virtual instrument for the measurement of the characteristics of magnetic materials', IEEE Trans. Instrum. Meas., 1992, 41, pp. 1005-1009

5 SCHNEIDER, II.R., and SCHWEDERSKI, T': 'Computer aided measurement of AC-hysteresis loops', IEEE Trans. Magn., 1984, MAG-20, pp. 1714-1716

6 BOGLIETTI, A., FERRARIS, P.M., and PROFUMO, F.: 'Iron losses in magnetic matcrials with six-step and PWM inverter supply', ILEE Trans, Magn., 1991, 27, pp. 5334.5336

7 CHEN, D.Y.: 'Comparison of high frequency magnetic core losses under two different driving conditions: a sinusoidal and a square-wave voltage'. Proccedings of IEEE PFSC'78, 1978, pp. 237-241

8 CARMINATI, E, and FERRERO, $A$.: 'Determination of the normal magnetisation curve and the total losses of magnetic materials by means of numerical techniques', Energ. Elettr., 1984, pp. 85-92

9 LO, Y.K., CHEN, C.L., LIN, J.S., and WU, J.Y.: 'A new high-performance $\mathrm{AC}-$ to- $\Lambda \mathrm{C}$ conversion system'. Proccedings of IFEE IECON'93, 1993, pp. 824829 